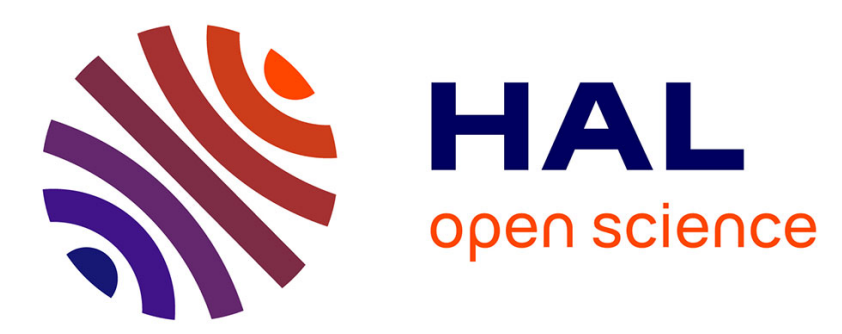

\title{
Vocal tract resonances in singing: variation with laryngeal mechanism for male operatic singers in chest and falsetto registers
}

\author{
Nathalie Henrich Bernardoni, John Smith, Joe Wolfe
}

\section{- To cite this version:}

Nathalie Henrich Bernardoni, John Smith, Joe Wolfe. Vocal tract resonances in singing: variation with laryngeal mechanism for male operatic singers in chest and falsetto registers. Journal of the Acoustical Society of America, 2014, 135 (1), pp.491-501. 10.1121/1.4836255 . hal-01056878

\section{HAL Id: hal-01056878 https://hal.science/hal-01056878}

Submitted on 20 Aug 2014

HAL is a multi-disciplinary open access archive for the deposit and dissemination of scientific research documents, whether they are published or not. The documents may come from teaching and research institutions in France or abroad, or from public or private research centers.
L'archive ouverte pluridisciplinaire HAL, est destinée au dépôt et à la diffusion de documents scientifiques de niveau recherche, publiés ou non, émanant des établissements d'enseignement et de recherche français ou étrangers, des laboratoires publics ou privés. 


\title{
Vocal tract resonances in singing: Variation with laryngeal mechanism for male operatic singers in chest and falsetto registers
}

\author{
Nathalie Henrich Bernardoni ${ }^{a}$ \\ Department of Speech and Cognition, GIPSA-lab (UMR5216: CNRS, Grenoble Institute of Technology, \\ Grenoble University), Grenoble, France \\ John Smith and Joe Wolfe \\ School of Physics, University of New South Wales, Sydney, New South Wales 2052, Australia
}

(Received 17 October 2012; revised 5 November 2013; accepted 12 November 2013)

\begin{abstract}
Seven male operatic singers sang the same notes and vowels in their chest and their falsetto registers, covering the overlap frequency range where two main laryngeal mechanisms can be identified by means of electroglottography: M1 in chest register and M2 in falsetto register. Glottal contact quotients determined using electroglottography were typically lower by 0.27 in M2 than in M1. Vocal tract resonance frequencies were measured by using broadband excitation at the lips and found to be typically lower in M2 than in M1 sung at the same pitch and vowel; $R 1$ typically by $65 \mathrm{~Hz}$ and $R 2$ by $90 \mathrm{~Hz}$. These shifts in tract resonances were only weakly correlated with the changes in the contact quotient or laryngeal height that were measured simultaneously. There was considerable variability in the resonance tuning strategies used by the singers, and no evidence of a uniform systematic tuning strategy used by all singers. A simple model estimates that the shifts in resonance frequencies are consistent with the effective glottal area in falsetto register (M2) being $60 \%-70 \%$ of its value in chest register (M1). (C) 2014 Acoustical Society of America.
\end{abstract}

[http://dx.doi.org/10.1121/1.4836255]

PACS number(s): 43.75.Rs, 43.70.Gr [CHS]

Pages: 491-501

\section{INTRODUCTION}

The source-filter model of voice production usually locates the source at the glottis and identifies the vocal tract as the filter. Resonances in the vocal tract produce maxima in the spectral envelopes of the output sound; these maxima are called formants. The source and filter interact and different tract configurations are understood to affect glottal behavior (Rothenberg, 1980; Fant and Lin, 1987; Childers and Wong, 1994; Barney et al., 2007; Titze, 2004, 2008). Changes in glottal configuration have been demonstrated to change the vocal tract resonance and formant frequencies in speech (Klatt and Klatt, 1990; Barney et al., 2007; Swerdlin et al., 2010) and their bandwidths (Nord et al., 1986). In singing, some research has focused on glottal properties and some on vocal tract acoustics and articulatory behaviors, but few studies have investigated the relationship between them (Miller and Schutte, 2005; Hanna et al., 2012). This paper investigates whether the vocal tract resonance frequencies change with the change in laryngeal mechanism associated with the chest-falsetto register transition. It reports simultaneous measurements of vocal fold vibration, using electroglottography, and vocal tract resonances, measured using broadband excitation at the lips.

Male operatic singers commonly use from one to three singing-voice registers to cover their working range (Henrich, 2006). Except in the case of the "voix mixte" register, these

\footnotetext{
a) Author to whom correspondence should be addressed. Electronic mail: Nathalie.Henrich@gipsa-lab.fr
}

singing-voice registers are usually associated with different timbres, and thus can often be distinguished by a trained listener. The timbral modifications may result from several aspects of the vocal gesture, including intrinsic differences in the laryngeal behavior, their impact on vocal-tract acoustics, and articulatory adjustments.

When the glottal biomechanics are considered, the following two main laryngeal mechanisms can be distinguished and identified by means of electroglottography: M1, in which modal or chest registers are produced, and M2, in which loft or falsetto registers are produced (Hollien, 1974; Roubeau, 1993; Henrich, 2006; Roubeau et al., 2009). (When the terms "modal" and "falsetto" are used to describe laryngeal mechanisms rather than specific singing-voice qualities, they are synonymous with M1 and M2.) Laryngeal mechanisms M1 and M2 are thought to be associated, respectively, with the increase or decrease in vibrating mass that results from the coupling or decoupling (respectively) of the layered vocal fold structure to the inferior thyro-arytenoid or vocalis muscle (Hirano, 1982). Counter-tenors and altos excepted, male operatic singers are trained to sing mainly in chest register, which is produced using M1. Even if it is not used in performance, some of them also practice their falsetto register, which is produced using M2. There is a range of frequencies where both laryngeal mechanisms are possible and the singer can choose to use either (Roubeau et al., 2004).

The use of a given laryngeal mechanism influences glottal-source parameters such as the open, closed or contact quotient (Henrich et al., 2005). The glottal contact quotient is positively correlated with sound pressure level (SPL) in 
M1, and with fundamental frequency in M2. It is generally less than $50 \%$ of the fundamental period in M2, while it can take higher values (as large as 70\%) in M1. At any given pitch within the overlap range, the contact quotient is commonly found to be higher in M1 than in M2. The vocal-fold vibratory amplitude also depends on the laryngeal mechanism in use, being greater in M1 than in M2 (Rubin and Hirt, 1960 as cited in Hollien, 1974; Hoppe et al., 2003). Barney et al. (2007) have demonstrated that an increase in glottal width or glottal-opening duration will raise the first formant frequency. It is thus possible that the observed differences in glottal behavior between the two main laryngeal mechanisms could be reflected in differences in the acoustics of the vocal tract. Several studies have assessed the sound spectral differences between singing-voice registers (Colton, 1972; Large et al., 1972; Hollien, 1974; Neumann et al., 2005). However the sound is a consequence of both the source spectrum at the vocal folds and the transfer characteristics of the vocal tract, and it is difficult to untangle their relative contributions using the sound spectrum alone.

Recently, dynamic magnetic resonance imaging (MRI) has been used to study articulatory adjustments associated with transitions between singing-voice registers (Echternach et al., 2008, 2009, 2010). These studies have shown that only minor changes in the vocal tract are associated with the transition between modal and falsetto registers in male and female voices. Larynx height and tilt were measured on MRI images using a proper metric. The tilt was derived as the angle between a line from the anterior commissure to the vocal process and a line connecting the cranial-most part of the dens axis and the caudo-anterior edge of the sixth vertebra. The larynx was found to be higher and more tilted in falsetto (M2) than in modal register (M1), and the tongue dorsum was lifted more in falsetto register.

The acoustic response of the vocal tract can also be measured non-invasively during a singing performance by means of a broadband acoustic source and a microphone placed at the lower lip (Epps et al., 1997). This introduces the complication that the tract is measured from the opposite end to where the vocal folds are located. It is also measured in parallel with the external radiation field, which means that, although the resonance frequencies can be determined, their bandwidths and magnitudes cannot. The changes in resonance frequency associated with changes in tract geometry in the supraglottal region can thus be reliably characterized (Joliveau et al., 2004a,b; Henrich et al., 2007; Garnier et al., 2010; Henrich et al., 2011). Changes in glottal characteristics alter the reflection condition at the upstream end of the supraglottal region and can thus alter the measured resonance frequencies; e.g., shifts in resonance frequencies between normal, creak, and whisper phonation have been measured by (Swerdlin et al., 2010). During phonation, although the glottal area is constantly varying, the measured impedance strongly resembles that measured by miming with the glottis closed, albeit with the expected slight shifts in resonance frequency due to the effective opening area of the glottis. Resonances typically involve multiple reflections and it might be expected that the effective glottal area is an average of the variations in glottal area with time. The exception is when the impedance is measured at a harmonic of the pitch frequency when the glottal area should remain constant, however, these are the only frequencies at which the impedance at the lips cannot be measured during phonation as the voice signal interferes with those frequency components of the injected signal. Thus the nonlinearities associated with vocal fold vibration do not affect the impedance measurements, except at these harmonics. The subglottal region also has resonances that might affect the measured impedance via the varying glottal opening during phonation. Experiments with a new technique (Hanna et al., 2012) have allowed the subglottal resonances to be discerned, but only when the folds are widely separated during respirationthey are not evident during normal phonation. This suggests that application of this technique to measure the differences in resonance frequencies between M1 and M2 phonation could provide an estimate of any change in effective area that might be involved, and that could be related to those observed via endoscopy.

In this paper, the resonance frequencies of the vocal tract are measured during phonation using broadband excitation at the lips. Compared with linear prediction or MRIreconstructed area functions, this method has the advantage of making no assumptions about glottal source properties. Compared with techniques requiring glottal-source adjustments, such as vocal fry or ingressive phonation (Miller et al., 1997), it has the advantage of allowing direct measurement during M1 and M2 phonation. Electroglottographic vocal-fold contact area and vocal-tract resonance frequencies were measured simultaneously on professional operatic tenors and baritones. Measurements included the overlap range where the singer can choose to use either laryngeal mechanism.

\section{MATERIALS AND METHODS}

\section{A. Resonance measurements}

Vocal tract resonances were measured at UNSW using a modified version of a technique described previously (Epps et al., 1997; Joliveau et al., 2004a). A computer (Macintosh IIci-Apple Computer, CA) with an analog interface card (National Instruments NB-A2100, Austin, TX) synthesizes a broadband signal as a sum of sine waves with frequencies spaced at $5.38 \mathrm{~Hz}$ and phases adjusted to improve the signal to noise ratio (Smith, 1995). This signal is amplified and drives an acoustically isolated loudspeaker connected via an exponential horn to a tube with inner diameter $7 \mathrm{~mm}$. This acoustic source is positioned at the subject's lower lip (Fig. 1). Attached to it is a prepolarized pressure-field microphone (DeltaTron Pressure-field 1/4 in. Microphone Type 4944A, Bruel \& Kjaer, Denmark). The acoustic source excites the vocal tract during singing, and both the singing voice and the response of the tract to the excitation signal are recorded by the microphone. The ratio of the latter signal to one measured previously on the outside of the closed lips gives the ratio of two impedances: the impedance of the tract in parallel with the radiation field and that of the radiation field itself. The frequencies of the vocal tract resonances are measured from this impedance ratio. 


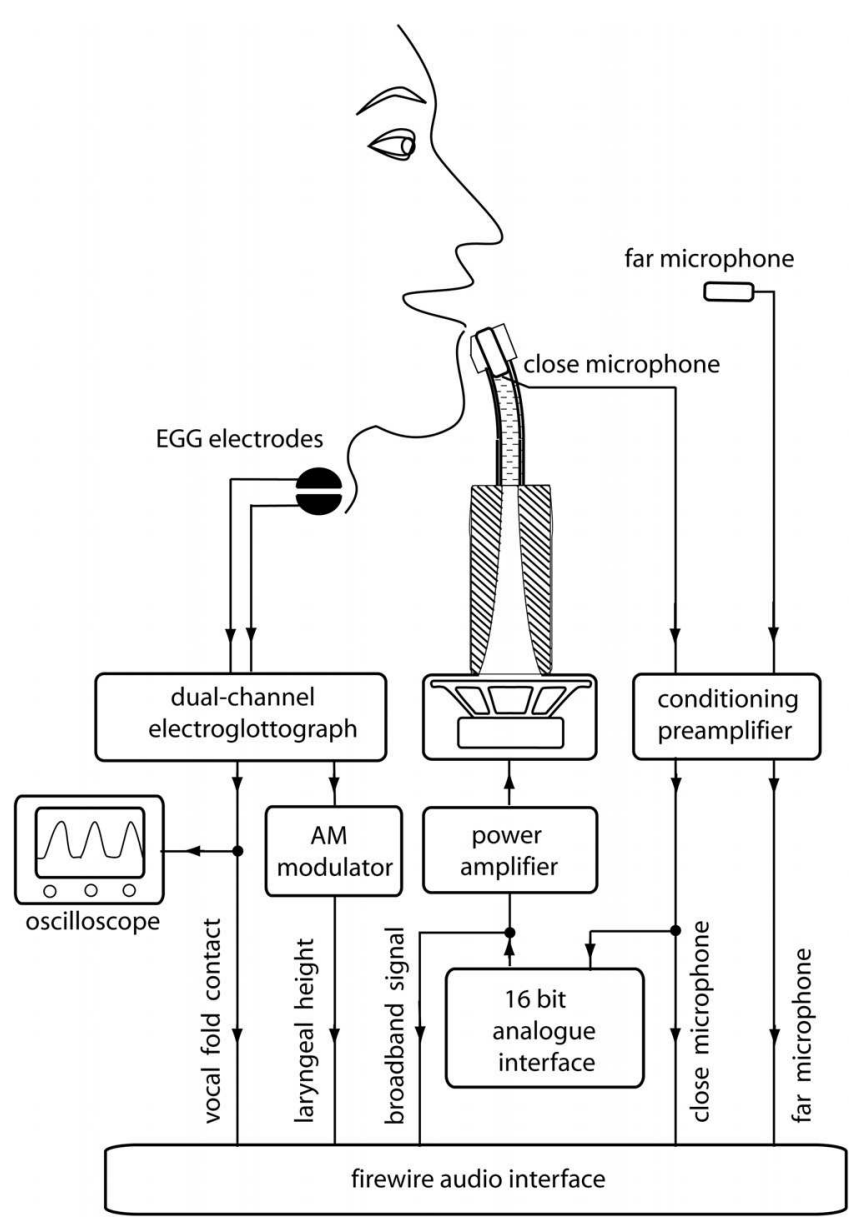

FIG. 1. Schematic diagram (not to scale) of the experimental apparatus.

\section{B. Electroglottographic measurements}

The vocal-fold contact area and laryngeal height were measured with a dual-channel electroglottograph (Glottal Enterprises, model EG-2). The electroglottographic (EGG) signal was monitored throughout the experiment on an oscilloscope (see Fig. 1). The laryngeal height signal provided by the EGG device is a direct current (DC) signal; consequently it was amplitude modulated at $395 \mathrm{~Hz}$ for recording and subsequently demodulated using MATLAB. Although the laryngeal height signal was calibrated for each subject, some calibrations were judged unreliable; consequently changes in laryngeal height were not compared between subjects.

The fundamental frequency and contact quotient were derived from the differentiated EGG signal (DEGG) using the correlation-based method for automatic measurement of glottal-source parameters (DECOM) described in Henrich et al. (2004). The singing-voice registers (chest or falsetto) were assessed by the singer himself during recording. The laryngeal mechanism (M1 or M2) was checked during the experiments by monitoring the EGG waveform and amplitude on an oscilloscope, and it was confirmed in a post-processing stage by comparing EGG waveforms and amplitudes at similar pitches.

\section{Recordings and analysis}

The close microphone used to measure resonances was placed at the subject's lower lip. A second microphone, also
Type 4944A, was placed at the level of the mouth and directly in front if it at a distance of about $70 \mathrm{~cm}$. The distance varied by a few centimeters from one singer to another, but for each singer was kept constant and was measured for the purpose of SPL measurements. Both microphone signals were amplified using a conditioning pre-amplifier (Nexus 2690, Bruel \& Kjaer, Denmark), and its internal reference signal ( $1 \mathrm{kHz}, 2 \mathrm{~V}$ peak-to-peak) was used for SPL calibration. The two audio signals (close and far microphone), the EGG and larynx tracking signals, and the synthetic broadband excitation signal were simultaneously recorded on five channels of a Firewire audio interface (MOTU 828, Cambridge, MA), sampled at $44.1 \mathrm{kHz}$ with 16 bit resolution. The measurements were conducted in a room with low background noise and low reverberation.

The excitation signal had a period of $93 \mathrm{~ms}\left(=2^{12}\right.$ samples $/ 44.1 \mathrm{kHz}$ ) and was maintained for 40 complete cycles lasting $3.7 \mathrm{~s}$. The first and last measured cycles were discarded and the remaining cycles were averaged before an impedance spectrum was calculated via a fast Fourier transform. The impedance ratio was calculated as the ratio between open-lip and closed-lip conditions. All the components of the broadband signal are harmonically related to the sampling frequency of the analog interface and so no windowing is required.

Using the close-microphone and broadband excitation signals, the impedance ratios described above were derived for each sustained spoken or sung sound. The resonance frequencies were measured by one author and checked by another. SPL was calculated using the far microphone signal. Fundamental frequency, glottal contact quotient and laryngeal height were derived from the EGG and larynx-tracking signals, as described above in Sec. II B. They were averaged for each sustained-sound sample during the time window when the mouth was externally excited.

\section{The subjects}

Table I presents details of the seven singers (two baritones and five tenors) who volunteered as subjects. All were professional or semi-professional Australian singers, five of them being employed full-time by a national opera company.

\section{E. Experimental protocol}

Three vowels, a subset of those used in previous studies (Joliveau et al., 2004b; Henrich et al., 2011), were chosen. The vowel to be sung was indicated by presenting a printed card showing the vowel in a carrier word: $h\langle$ vowel $\rangle d$ [i.e., hard, hoard, and heard, which are typically pronounced /hæd/, /hod/, and /h3d/ in Australia (Delbridge, 1985)].

Subjects were asked to sing the target note, the note below it on the $\mathrm{C}$ major scale and then the target note again. After the third note started, an experimenter started the resonance measurement, and the subject sustained the note until after the broadband sound stopped. The three notes were produced legato, without intervening silence or consonant. Resonance measurements were made during the 
TABLE I. Details of the participating singers. The overlap range indicates the region where both M1 and M2 were studied for that singer. Experience and singing style are specified using the taxonomy of Bunch and Chapman (2000) where the integer part indicates the professional level (e.g., $2=$ international, $3=$ national, $5=$ local community) and the fractional part indicates the style (e.g., $3.1=$ national opera, $3.4=$ national concert-oratorio-recital, etc.).

\begin{tabular}{|c|c|c|c|c|c|c|c|}
\hline Singer & $\begin{array}{c}\text { Baritone } \\
\text { B1 }\end{array}$ & $\begin{array}{c}\text { Baritone } \\
\text { B2 }\end{array}$ & $\begin{array}{c}\text { Tenor } \\
\text { T1 }\end{array}$ & $\begin{array}{l}\text { Tenor } \\
\text { T2 }\end{array}$ & $\begin{array}{l}\text { Tenor } \\
\text { T3 }\end{array}$ & $\begin{array}{c}\text { Tenor } \\
\text { T4 }\end{array}$ & $\begin{array}{l}\text { Tenor } \\
\text { T5 }\end{array}$ \\
\hline Age & 32 & 34 & 35 & 42 & 33 & 60 & 22 \\
\hline Taxonomy & $2.15,3.4$ & 5.3 & 3.4 & 5.4 & $3.1 \mathrm{c}$ & $3.1 \mathrm{c}$ & $3.2 b, 3.4,3.9,3.15 a, 3.17$ \\
\hline Pitch overlap range & D4-G4 & A3-F4 & D4-B4 & D4-A4 & E3-A4 & A3-F4 & C4-A4 \\
\hline Frequency overlap range $(\mathrm{Hz})$ & $290-400$ & $220-350$ & $290-500$ & $290-440$ & $160-440$ & $220-350$ & $260-440$ \\
\hline
\end{tabular}

third note, so that for that note the sound file contained successively voice alone, voice plus broadband signal and voice alone. The target pitch was indicated by playing a note on a glockenspiel. For each vowel, each note in the $\mathrm{C}$ major scale was presented from the bottom to the top of each singer's self-described comfortable singing range. Where the ranges of the two laryngeal mechanisms M1 and M2 overlapped, each vowel-note combination was measured in each mechanism. After each vowel on each note was sung, they were also asked to speak the carrier word, but to sustain the vowel for about $4 \mathrm{~s}$ while a measurement was made.

\section{RESULTS AND DISCUSSION}

\section{A. Glottal behavior}

Figure 2 shows an example of how the measured parameters glottal contact quotient (CQ), SPL, and resonance frequencies $(R 1$ and $R 2)$ varied with frequency $f_{0}$ for singer B2. CQ is seen to be distinctly higher in chest (M1) than in falsetto register (M2). Although there is variability among singers, CQ in M2 was always smaller than in M1 when pairs of measurements were compared at the same pitch for the same singer and vowel (see Fig. 3). This is similar to the decrease in closed quotient estimated by inverse filtering when tenors
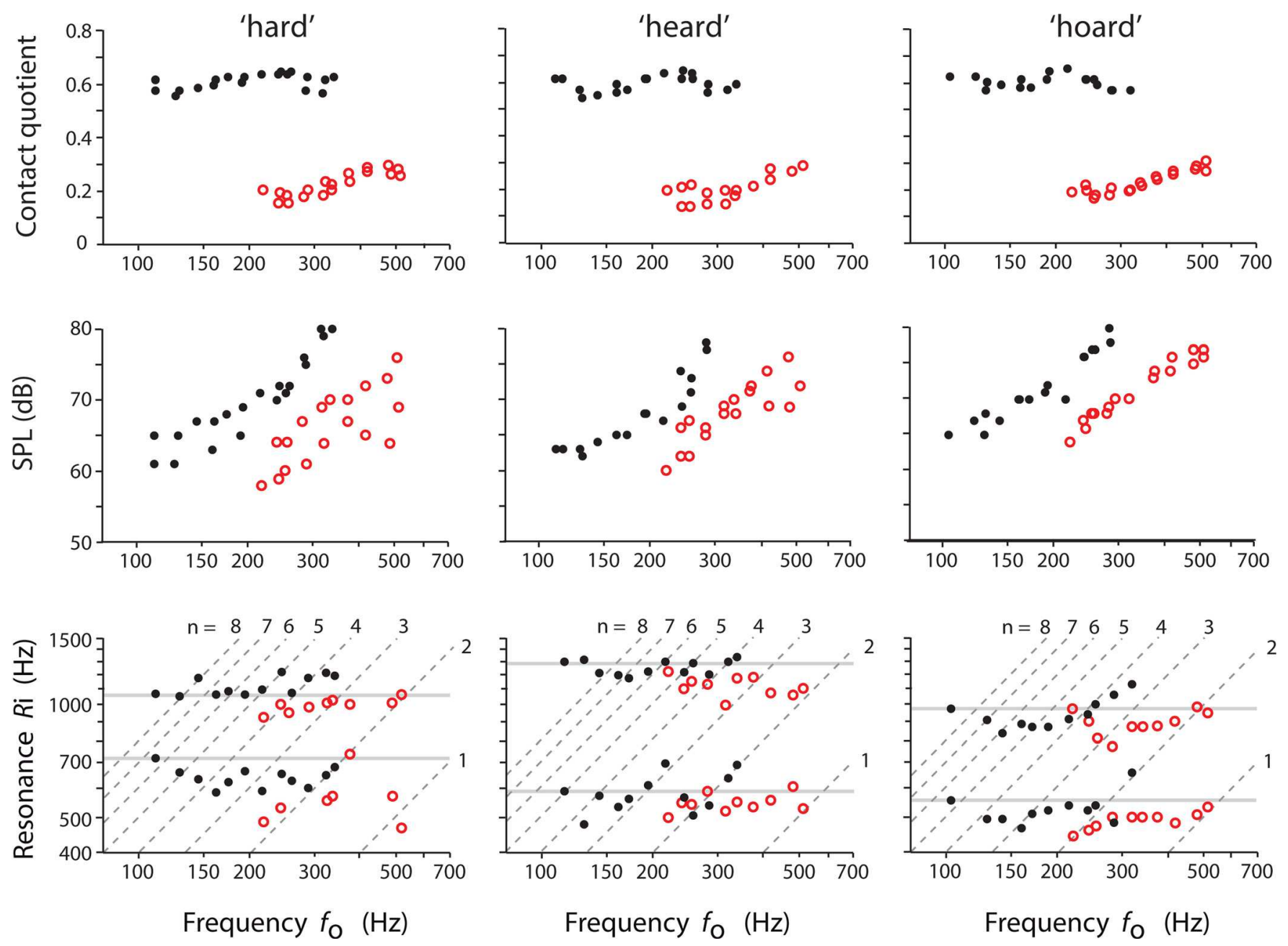

FIG. 2. (Color online) The variation of the CQ, SPL, and the first and second resonance frequencies $(R 1$ and $R 2)$ with the pitch frequency $f_{0}$ for baritone $\mathrm{B} 2$ singing in chest register with laryngeal mechanism M1 (closed circles) and falsetto with M2 (open circles). The diagonal, dashed gray lines indicate when a resonance frequency would coincide with the $n$th harmonic $\left(n f_{0}\right)$; i.e., the possible relationships $R \mathrm{i}=n f_{0}$. The horizontal gray lines indicate the resonance frequencies measured in speech for the same singer and vowel. 


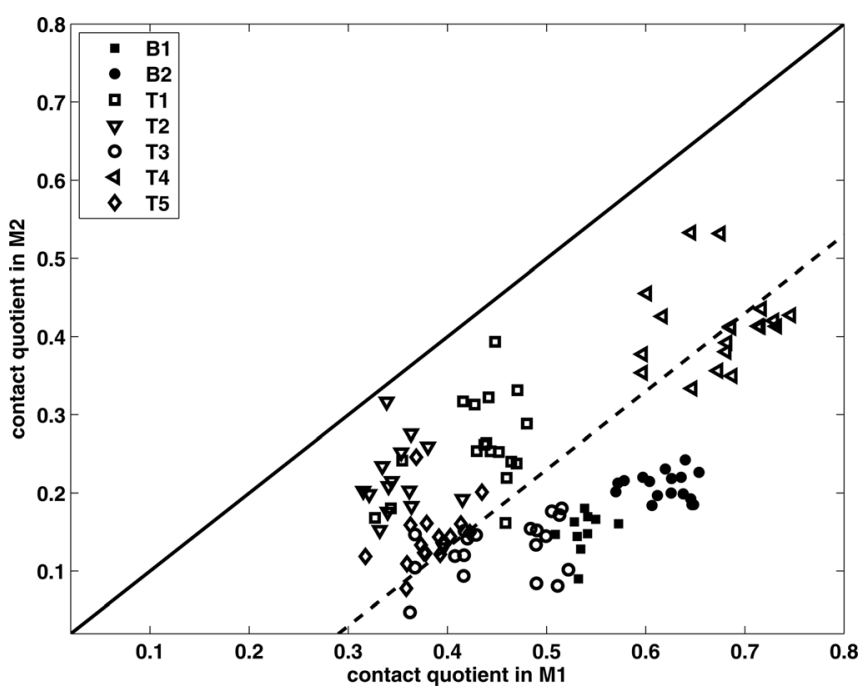

FIG. 3. The CQ for singing in the falsetto register with laryngeal mechanism M2 vs that for singing in chest register with mechanism M1, measured for the same singer, pitch, and vowel. Different symbols represent different singers; $\mathrm{B}$ indicates baritones and $\mathrm{T}$ tenors. The solid line indicates the relationship $\mathrm{CQ}_{\mathrm{M} 2}=\mathrm{CQ}_{\mathrm{M} 1}$ and the dashed line indicates their average measured relationship, i.e., $\mathrm{CQ}_{\mathrm{M} 2}=\mathrm{CQ}_{\mathrm{M} 1}-0.27$.

and baritones changed from modal to falsetto register (Sundberg and Högset, 2001; Salomão and Sundberg, 2009). Table II indicates that the contact quotient for all vowels in M1 exceeds that in M2 by an average value of $0.27 \pm 0.11$, in agreement with Henrich et al. (2005). A paired $t$-test indicated that the difference between the two laryngeal mechanisms in the overlap region was significant at the $0.01 \%$ level or lower for all vowels.

Table II also presents the measured contact quotient for vowels produced outside the overlap region. In chest register (M1) there was no statistically significant difference between CQ measured below or inside the overlap region. Similarly CQ in falsetto register (M2) was not significantly different when measured within or above the overlap region.

This study concentrated mainly on the effects of varying $f_{0}$, not those of varying SPL. However, because it is known that CQ can depend upon both $f_{0}$ and SPL (Henrich et al., 2005), the separate dependences upon $f_{0}$ and SPL were determined by fitting a multiple linear regression to the data. The results over the range studied here $(100-600 \mathrm{~Hz}$ and 52-83 dB) varied from singer to singer. In mechanism M1, statistically significant (at the $0.1 \%$ level) variations in CQ with increasing $f_{0}$ were small, with an average slope of only $0.09 \mathrm{kHz}^{-1}$. In a previous study, Henrich et al. (2005) found no strong correlations between CQ and $f_{0}$ in M1. A linear relationship was found between the contact quotient $\mathrm{CQ}$ and SPL with a slope of $0.015 \pm 0.001 \mathrm{~dB}^{-1}\left(r^{2}=0.30\right)$; this is also consistent with the results of Henrich et al. (2005) for tenors and baritones.

In mechanism M2, a statistically significant positive correlation between $\mathrm{CQ}$ and $f_{0}$ (at the $0.01 \%$ ) level was found for the two baritones. This is consistent with earlier studies on counter-tenors (Henrich et al., 2005). However the tenors showed no significant correlation between CQ and $f_{0}$. The absence of any significant observed correlations between CQ and SPL in M2 is consistent with Henrich et al. (2005).

The laryngeal height measured by the electroglottograph is an empirical quantity determined from the signals measured above and below the larynx, and correlated with larynx height. Reliable calibrations were only available for four of the seven subjects, so detailed comparisons could not be made between subjects. The change in this parameter when the laryngeal mechanism changed from M1 to M2 varied from singer to singer. When comparisons were made between M1 and M2 at the same pitch for the same singer and vowel, the laryngeal height was found to increase significantly (at the 1\% level) for 4 singers (B1, B2, T3, T4), to decrease significantly (at the $0.01 \%$ level) for two singers (T1, T5), and to be not significantly different for one singer (T2). A small average rise in laryngeal position between productions in chest and in falsetto registers is consistent with the minor elevation and tilting of the larynx observed by Echternach et al. (2008, 2009, 2010) using dynamic MRI on professional operatic tenors.

TABLE II. The measured values of the CQ for singers in chest register with laryngeal mechanism M1 and falsetto with M2. Values are also given for the overlap region where a singer could use either mechanism. $\triangle \mathrm{CQ}$ denotes the difference between pairs of values of CQ for mechanisms M1 and M2 that were measured for the same pitch, singer, and vowel in the overlap region. In this and subsequent tables, and the text, the results are presented as mean \pm standard deviation (number of samples).

\begin{tabular}{|c|c|c|c|c|}
\hline Vowel & & Below overlap & Overlap region & Above overlap \\
\hline \multirow[t]{3}{*}{ Hard } & M1 & $0.47 \pm 0.09(76)$ & $0.50 \pm 0.12(73)$ & - \\
\hline & M2 & - & $0.22 \pm 0.10(74)$ & $0.24 \pm 0.05$ \\
\hline & $\Delta \mathrm{CQ}$ & - & $0.28 \pm 0.10$ & - \\
\hline \multirow[t]{3}{*}{ Heard } & M1 & $0.48 \pm 0.10(81)$ & $0.47 \pm 0.12(72)$ & - \\
\hline & M2 & - & $0.22 \pm 0.10(65)$ & $0.25 \pm 0.06$ \\
\hline & $\Delta \mathrm{CQ}$ & - & $0.27 \pm 0.11(49)$ & - \\
\hline \multirow[t]{3}{*}{ Hoard } & M1 & $0.49 \pm 0.10(78)$ & $0.48 \pm 0.12(66)$ & - \\
\hline & M2 & - & $0.22 \pm 0.11(61)$ & $0.23 \pm 0.06(20)$ \\
\hline & $\Delta \mathrm{CQ}$ & - & $0.27 \pm 0.10(53)$ & - \\
\hline \multirow[t]{3}{*}{ All vowels } & M1 & $0.48 \pm 0.10(235)$ & $0.49 \pm 0.12(211)$ & - \\
\hline & M2 & - & $0.22 \pm 0.10(200)$ & $0.24 \pm 0.06$ \\
\hline & $\Delta \mathrm{CQ}$ & - & $0.27 \pm 0.11$ & - \\
\hline
\end{tabular}


TABLE III. The measured values of the first $(R 1)$ and second $(R 2)$ vocal tract resonances for vowels produced by singers in chest register with laryngeal mechanism M1 and falsetto register with M2. $\Delta R$ i denotes the frequency by which $R \mathrm{i}$ in M1 exceeded that in M2 measured between pairs of values of $R \mathrm{i}$ measured for the same pitch, singer, and vowel. The symbol * indicates that the difference $\Delta R$ i was significant at the $1 \%$ level or lower as indicated by a paired $t$-test.

\begin{tabular}{|c|c|c|c|c|c|}
\hline & \multirow[b]{2}{*}{ Mechanism } & \multicolumn{4}{|c|}{ Vowel } \\
\hline & & Region & Hard & Heard & Hoard \\
\hline$R 1(\mathrm{~Hz})$ & M1 & below overlap & $645 \pm 65(45)$ & $555 \pm 65(40)$ & $515 \pm 60(42)$ \\
\hline$R 1(\mathrm{~Hz})$ & M1 & overlap & $645 \pm 70(32)$ & $585 \pm 85(38)$ & $580 \pm 70(37)$ \\
\hline$\Delta R 1(\mathrm{~Hz})$ & M1-M2 & overlap & $75 \pm 80(32)^{*}$ & $50 \pm 70(38)^{*}$ & $75 \pm 70(37)^{*}$ \\
\hline$R 1(\mathrm{~Hz})$ & M2 & overlap & $570 \pm 70(32)$ & $535 \pm 40(38)$ & $505 \pm 50(37)$ \\
\hline$R 1(\mathrm{~Hz})$ & M2 & above overlap & $623 \pm 125(10)$ & $510 \pm 70(10)$ & $490 \pm 55(10)$ \\
\hline$R 2(\mathrm{~Hz})$ & M1 & below overlap & $1080 \pm 70(40)$ & $1205 \pm 85(45)$ & $850 \pm 85(41)$ \\
\hline$R 2(\mathrm{~Hz})$ & M1 & overlap & $1105 \pm 75$ & $1230 \pm 75(32)$ & $1005 \pm 85(36)$ \\
\hline$\Delta R 2(\mathrm{~Hz})$ & M1-M2 & overlap & $85 \pm 110(43)^{*}$ & $70 \pm 95(32)^{*}$ & $110 \pm 105(36)$ \\
\hline$R 2(\mathrm{~Hz})$ & M2 & overlap & $1020 \pm 85(43)$ & $1160 \pm 55(32)$ & $900 \pm 85(36)$ \\
\hline$R 2(\mathrm{~Hz})$ & M2 & above overlap & $1035 \pm 75(9)$ & $1090 \pm 85(10)$ & $940 \pm 85(12)$ \\
\hline
\end{tabular}

The measured variation of laryngeal height with pitch varied considerably between singers. In chest register, the only significant (at the $0.3 \%$ level) correlations were for three singers (T1, T2, and T5), where the laryngeal height increased with increasing pitch for all three vowels. In falsetto register, only one of the 21 possible combinations of singer and vowel indicated a significant increase in laryngeal height with increasing pitch, whereas in three combinations the laryngeal height decreased.

\section{B. Vocal tract resonances}

Figure 2 shows an example of how the measured resonance frequencies varied with frequency $f_{0}$ for singer B2. The values of $R 1$ and $R 2$ are seen to be generally lower in $\mathrm{M} 2$ (falsetto productions) than in M1 (chest productions). Although there is variation among singers, the average values of $R 1$ and $R 2$ are typically around $60-70 \mathrm{~Hz}$ lower in $\mathrm{M} 2$ than they are in M1-see Table III. This difference can be further characterized by calculating $\Delta R 1$ and $\Delta R 2$, which are the amounts by which $R 1$ and $R 2$ measured in M1 exceed that in $\mathrm{M} 2$, when measured for the same singer, vowel, and pitch. The differences $\Delta R 1$ and $\Delta R 2$ are each statistically significant at the $0.01 \%$ level or better. Figure 4(a) shows the values of $R 1$ in M1 plotted against $R 1$ in $\mathrm{M} 2$ when measured for the same singer, vowel, and pitch. A similar plot [Fig. 4(b)] demonstrates how $R 2$ varies between vowels produced in the two laryngeal mechanisms. The average ratio of these values in M2 with respect to those in M1 was $0.90 \pm 0.12$ (107) for $R 1$ and $0.92 \pm 0.10$ (111) for $R 2$. Tenor T5 was the only singer whose $R 1$ and $R 2$ values were consistently higher in M2 than M1. The measured overall decrease in resonance frequencies on the transition from chest register in laryngeal mechanism M1 to falsetto register in M2 is the opposite from that reported for the transition from "classical" to "non-classical" productions (Sundberg et al., 2011).

Although Table III indicates that the average values of $R 1$ and $R 2$ in M1 measured in the overlap region were typically slightly higher than their values measured below the overlap region, these differences were not significant at the $1 \%$ level. Similarly, although the values of $R 1$ and $R 2$ in M2 were slightly lower when measured in the overlap region than when measured above, these differences were again not significant at the $1 \%$ level. The exception was for the vowel in "hoard" where the increase in the values of $R 1$ and $R 2$ in
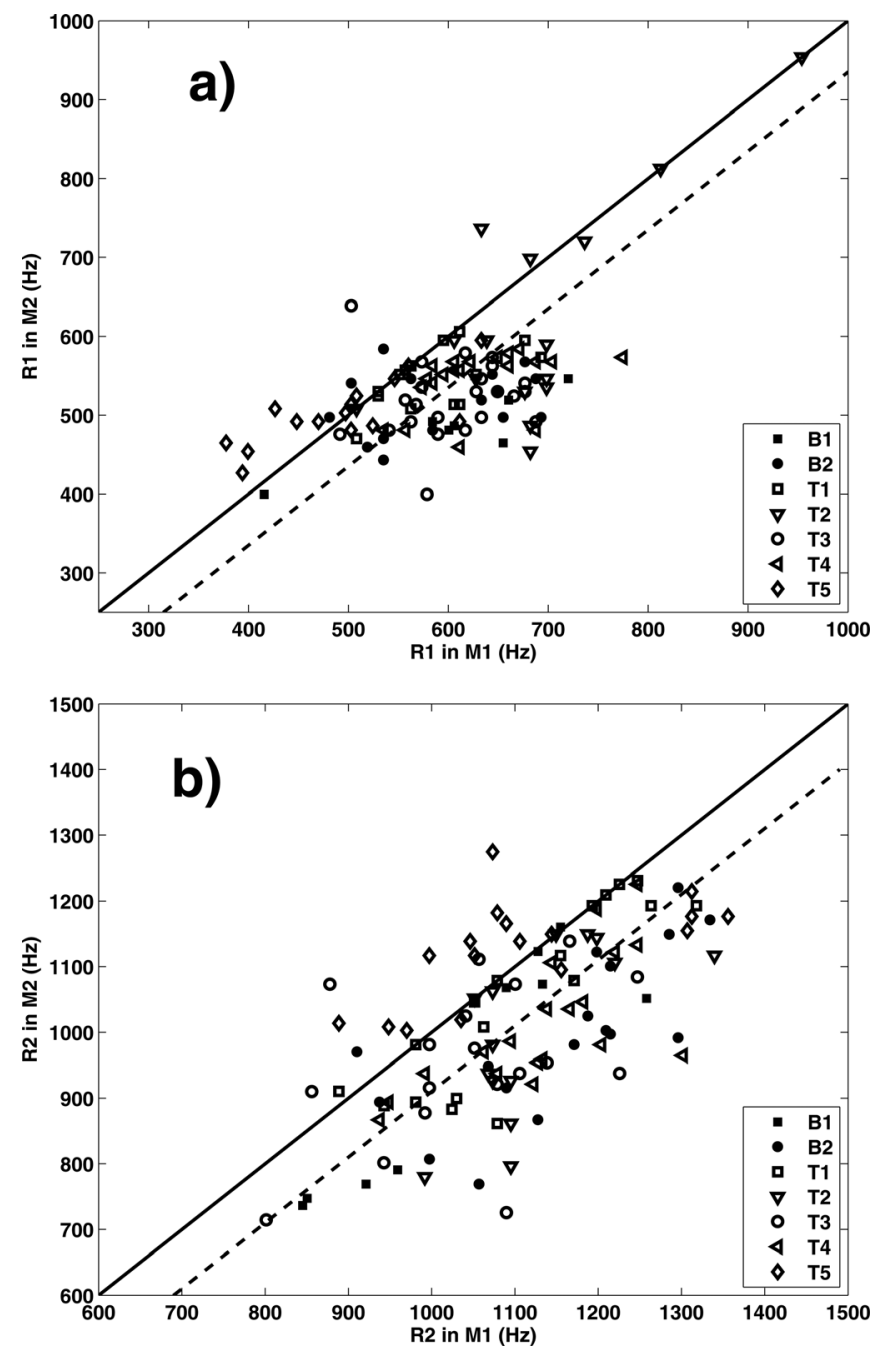

FIG. 4. (a) Shows plots of the first tract resonance frequency, $R 1$, for singing in the falsetto register with laryngeal mechanism M2 vs $R 1$ for singing in chest register with mechanism M1, measured for the same singer, pitch, and vowel. (b) Shows the same for the second tract resonance, $R 2$. The solid gray line indicates the relationship $R \mathrm{i}_{\mathrm{M} 2}=R \mathrm{i}_{\mathrm{M} 1}$, and the dashed lines indicate the average measured relationships $R 1_{\mathrm{M} 2}=R 1_{\mathrm{M} 1}-65 \mathrm{~Hz}$ and $R 2_{\mathrm{M} 2}=R 2_{\mathrm{M} 1}-90 \mathrm{~Hz}$. 
M1 when in the overlap region was significant at the $0.01 \%$ level. This is possibly because the values of $R 1$ and $R 2$ in speech for "hoard" are lower than in "hard" and "heard," and thus resonance tuning, which usually involves an increase in resonance frequencies, might become increasingly advantageous as the frequency increases-see Fig. 2. There thus appears to be nothing special about the tract resonances in the overlap region that allow a singer to use chest register with laryngeal mechanism M1 or falsetto register with laryngeal mechanism M2.

The differences $\Delta R 1$ and $\Delta R 2$ from 34 measurements for the same singer, pitch, and vowel were found to be correlated $\left(r^{2}=0.62\right)$ and described by $\Delta R 2=60 \mathrm{~Hz}+0.71 \times \Delta R 1$ and significant at the $0.1 \%$ level. This suggests that the changes in resonance frequency between chest and falsetto registers might share a common origin.

\section{Are the resonances tuned to match harmonics?}

As the fundamental frequency $f_{0}$ increases it is likely that $R 1$ and $R 2$, even if left unaltered from their values in speech, will at times approach a harmonic. Reliable identification of resonance tuning, which can be defined as the adjustment of a resonance so that it becomes closer in frequency to $f_{0}$ or a harmonic, generally requires several successive values of $f_{0}$ to be tuned closed to the same harmonic. Figure 2 plots $R 1$ and $R 2$ as a function of $f_{0}$ for all three vowels produced in the two laryngeal mechanisms for the baritone B2. Even at the highest pitches reached in M1, the fundamental frequency $f_{0}$ was less than the value of $R 1$ measured in speech for that singer and vowel, and consequently the strategy of tuning $R 1$ to $f_{0}\left(R 1: f_{0}\right.$ tuning) over a wide pitch range that has been observed at higher pitches for most female singers is absent (Sundberg, 1975; Joliveau et al., 2004a,b; Garnier et al., 2010; Henrich et al., 2011). There is, however, an indication of the onset of $R 1: f_{0}$ tuning at the highest frequencies in M2 for "hoard." There is also evidence of $R 1: 2 f_{0}$ and $R 1: 3 f_{0}$ tuning in M1, and evidence of $R 1: 2 f_{0}$ tuning in M2; strategies shown previously to be used by altos, tenors, and baritones (Henrich et al., 2011). For $R 2$, there are possible instances of $R 2: 4 f_{0}, R 2: 5 f_{0}, R 2: 6 f_{0}$, and $R 2: 8 f_{0}$ tuning in M1, but no clear instances in M2. Figure 5 shows some clear examples of $R 1: 3 f_{0}$ tuning in $\mathrm{M} 1$ for singer
T2. Although these examples of resonance tuning were evident, there was no single tuning strategy used by all singers, and no single singer used a consistent strategy for all vowels. Most of the singers in the present study differed from those of Sundberg et al. (2011) whose male operatic singers showed little evidence of resonance tuning over successive notes. The present results and those of Sundberg et al. also differ from those of Neumann et al. (2005) who, using audio spectra and ingressive phonation, concluded that (in the notation of this paper) $R 1: 2 f_{0}$ and $R 2: 4 f_{0}$ tuning were present in chest register (presumably in M1).

Why is there so much variation among this and previous reports - and among the subjects in this study? As reported earlier (Henrich et al., 2011), although some or all of the above resonance tuning strategies are used by most singers in the low voice categories (i.e., tenor and baritone), the frequency range over which each is employed can vary considerably. To illustrate this, Fig. 6 shows the combined data for all singers and vowels. The absence of obvious regimes of uniform resonance tuning indicates that the strategies, where present, vary considerably from singer to singer, unlike the situation for sopranos (e.g., see Fig. 2 in Henrich et al., 2011).

To investigate further the extent of resonance tuning, Fig. 7 shows the difference in frequency between the nearest harmonic of $f_{0}$ and $R 1$ or $R 2$ in the overlap region where both M1 and M2 are measured. None of these distributions show a strong maximum around zero, which would have indicated a high degree of tuning similar to that exhibited by sopranos at high pitch—for example see Fig. 8 in Henrich et al. (2011). The maximum in the distribution for $R 1$ is found around $20-40 \mathrm{~Hz}$ above the closest harmonic in M1 and slightly higher in M2 $(40-60 \mathrm{~Hz})$. The maximum in the distributions for $\mathrm{R} 2$ is found around $20-40 \mathrm{~Hz}$ above the closest harmonic for both M1 and M2.

The resonance behaviors observed in falsetto register here were measured for singers trained in the professional use of M1 and cannot simply be compared with those of male altos, who are trained in the professional use of M2.

\section{Is the phase of the acoustic load important?}

One possibility is that the phase of the acoustic load presented to the glottis might be important; e.g. some models
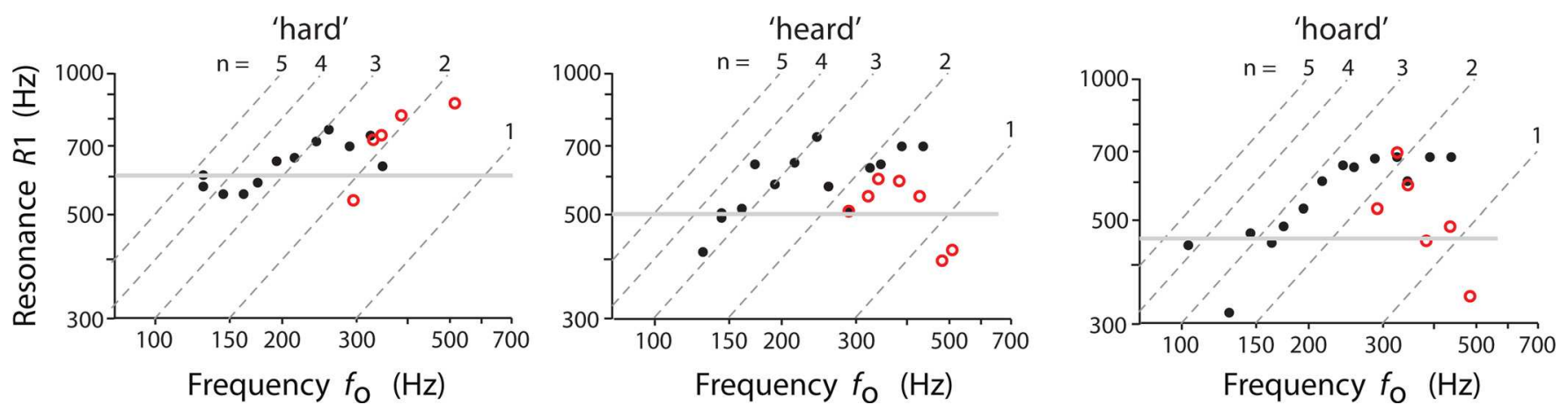

FIG. 5. (Color online) The variation of the first resonance frequency $(R 1)$ with the pitch frequency $f_{0}$ for tenor T2 singing in chest register with laryngeal mechanism M1 (closed circles) and in falsetto register with laryngeal mechanism M2 (open circles). The diagonal, dashed gray lines indicate when a resonance frequency would coincide with the nth harmonic $\left(n f_{0}\right)$; i.e., the possible relationships $R \mathrm{i}=n f_{0}$. The horizontal gray lines indicate the resonance frequencies measured in speech for the same singer and vowel. 

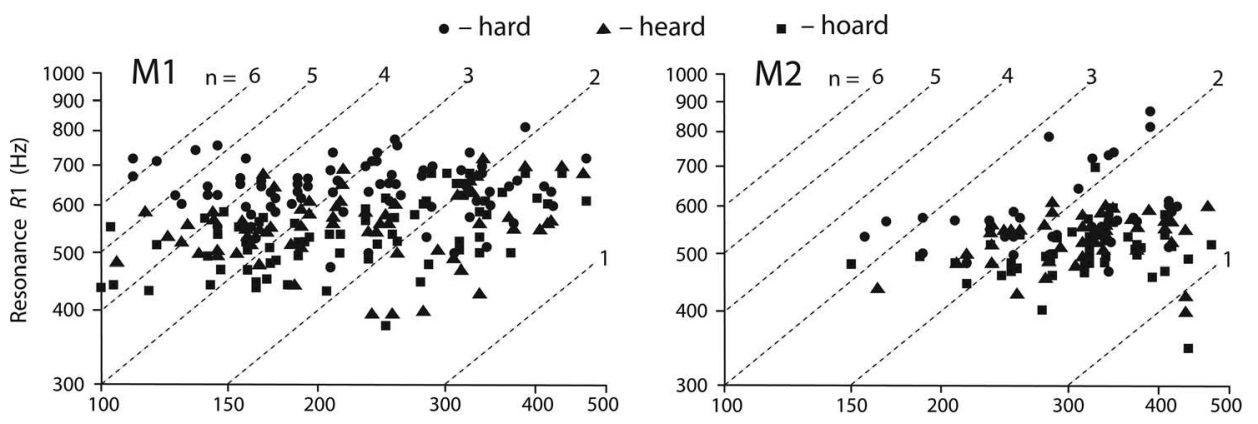

FIG. 6. The variation of the first and second resonance frequencies $(R 1$ and $R 2$ ) with the pitch frequency $f_{0}$ for all the singers in this study in mechanisms M1 and M2. Data from 741 measurements on seven singers (five tenors and two baritones) on three sustained vow-
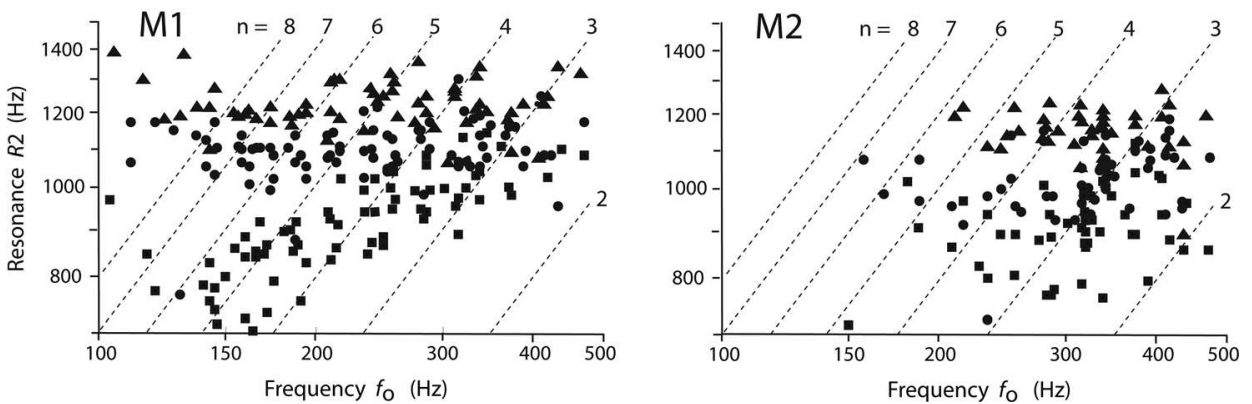
els (hard, heard, and hoard). The diagonal, dashed gray lines indicate when a resonance frequency would coincide with the $n$th harmonic $\left(n f_{0}\right)$; i.e., the possible relationships $R \mathrm{i}=n f_{0}$.

suggest that vocal fold vibration will be enhanced and more stable if the downstream load is inertive. In nearly all instances in this study, $f_{0}$ lay below $R 1$, as is usually the case for male singers. So, at the fundamental frequency of glottal vibration, the downstream acoustic load presented by the vocal tract at the glottis is inertive.

It is possible that, especially for loud phonations, the phase of the acoustic load at the second or perhaps higher harmonics could influence the vibration of the vocal folds. In the acoustic impedance spectrum of the load presented by the vocal tract at the glottis, the maxima and minima are not equally spaced: each minimum falls at a frequency close to and above that of a maximum-see Titze (2008); Wolfe et al. (2009). The downstream load is thus expected usually to be inertive, except if a tract resonance occurs just below the pitch frequency. If an inertive load were strongly preferred in the higher harmonics, one might expect that singers would avoid using values of $R 1$ and $R 2$ that were only slightly lower in frequency than $f_{0}$ or one of its harmonics.
The histograms presented in Fig. 7, however, show only a small reluctance to tune resonances slightly below one of the harmonics $n f_{0}$ at the sound levels of this experiment. Another possibility is that the shift between chest register in M1 and falsetto register in M2 could be associated with matching $R 1$ and/or $R 2$ to a different harmonic. In the overlap region for which there were data for M1 and M2 measured at the same $f_{0}$, the harmonic closest to $R 1$ remained the same for $74 \%$ of 106 measurements and it remained the same for $66 \%$ of 111 measurements of $R 2$. Because the $R \mathrm{i}$ are lower for $\mathrm{M} 2$, the resonances were closer to a lower harmonic in M2 than in M1 for 23\% and 31\% of measurements of $R 1$ and $R 2$, respectively. $R 1$ and $R 2$ were closer to a higher harmonic in M2 than in M1 for only 3\% and $4 \%$ of measurements, respectively, in the overlap range. It thus appears that the transition between chest register in mechanism M1 and falsetto register in M2 is not usually associated with changing the articulation to match a resonance to a different harmonic.
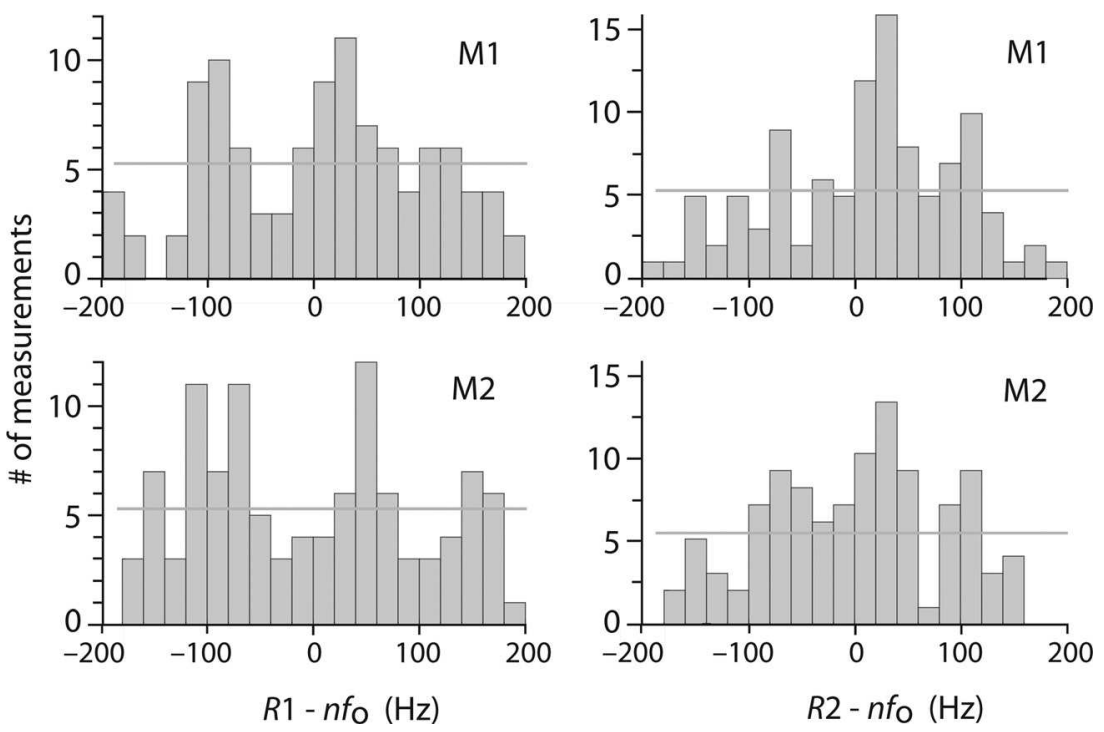

FIG. 7. Histograms showing the distribution of $R 1$ (106 measurements) and $R 2$ (111 measurements) about the closest harmonic of $f_{0}$. The presented data are from the overlap region where productions in both chest register (M1) and falsetto register (M2) were measured. The gray horizontal lines indicate the average. 


\section{E. What produces the changes in resonance frequency?}

There are several possibilities that might account for the systematic decrease in resonance frequencies between vowels sung in chest register using M1 and those sung in falsetto register using $\mathrm{M} 2$

(i) Changes in glottal-opening duration and glottal vibratory amplitude: Barney et al. (2007) have argued both theoretically, and using a time-varying mechanical model of a rectangular glottis coupled to a rigid vocal tract, that an increase in glottal width or an increase in open quotient (equivalent to a decrease in contact quotient) leads to an increase in $R 1$. In the present paper, the changes in $R 1$ and $R 2$ were never strongly correlated with the changes in CQ measured for the same singer, pitch, and vowel. However, glottal vibratory amplitude, i.e., the maximum range of glottal width change, has been found to be much wider in chest register than in falsetto (Rubin and Hirt, 1960 as cited in Hollien, 1974; Hoppe et al., 2003) and this may be a reason for the increase in $R 1$ between M2 and M1.

(ii) Changes in laryngeal height: $\mathrm{A}$ rise in laryngeal height shortens the vocal tract, and would therefore be expected to increase $R 1$ and $R 2$ (Sundberg and Nordström, 1983). A 1-cm rise in laryngeal height in a tract of $16.5 \mathrm{~cm}$ effective length would, in a simple model, be expected to produce an increase in $R 1$ on the order of several percent. However, in our measurements, the changes in $R 1$ and $R 2$ were not significantly correlated (at the $1 \%$ level) with changes in laryngeal height.

(iii) Change in mouth opening and tongue shape: A small, but measurable decrease in jaw opening could be expected to produce a decrease in $R 1$. However, the mouth opening was not measured in this study.

(iv) Change in glottal area: The values of $R 1$ and $R 2$ are higher in M1 compared with M2 and this would be consistent with a larger effective area in M1 if this were the only geometrical change produced by the change in mechanism.

At first one might expect that the longer duration of the measured no-contact phase in M2 would produce a larger effective glottal area. However, the geometry of the openings are different: In M1, the glottis usually uses less than the full length of the vocal folds, but the opening between them is relatively large. The glottal-area values measured on a 31-yr-old male singer by Hoppe et al. are around $32 \mathrm{~mm}^{2}$. In M2, the glottis typically has an increased length. Hoppe et al. (2003) found an increase in glottal-length from 14 to $17 \mathrm{~mm}$ during an ascending glissando. The glottis is also open for a longer fraction of the period. However, the glottal area in M2 is much smaller than that in M1 (around $20 \mathrm{~mm}^{2}$ in Hoppe et al., 2003). Also, a long narrow slot has a smaller inertance than a circle with the same area because its end effect is shorter (Cremer, 1984).

A simple model described by Swerdlin et al. (2010) gives analytical expressions for the frequency shifts produced by different glottal geometries. The model treats the vocal tract as a cylinder terminated at the lips by a baffled radiation field and at the other end by a supraglottal constriction and a glottis. Effective lengths and (uniform) cross sections are ascribed to each. (When M2 involves glottal leakage, that leakage would be included in the effective cross section, averaged across one cycle.) Because it is an approximate model and because only frequencies are calculated, wall losses are neglected. If it is assumed that the rest of the geometry is unchanged, the effective change in glottal area between different glottal mechanisms can be determined from the change in resonance frequency.

Thus if $R 1_{\mathrm{M} 1}$ is the value of $R 1$ measured for mechanism M1, the effective length of the tract, denoted by $L_{\mathrm{T}}$, can be calculated using

$$
L_{\mathrm{T}}=\tan ^{-1}\left(-k_{1} r_{\mathrm{T}}^{2} L_{\mathrm{G} 1} / r_{\mathrm{G} 1}^{2}\right) / k_{1}
$$

where $k_{1}=2 \pi R 1_{\mathrm{M} 1} / c$. This requires the assumption of values for $r_{\mathrm{T}}$, the radius of the tract, $L_{\mathrm{G} 1}$ the length of the glottis in M1 and also $r_{\mathrm{G} 1}$, the effective glottal radius in M1. Providing that $L_{\mathrm{T}}$ does not change between M1 and M2, then $r_{\mathrm{G} 2}$, the glottal radius in mechanism $\mathrm{M} 2$, can be calculated from $R 1_{\mathrm{M} 2}$, the value of $R 1$ measured for mechanism M2 using

$$
r_{\mathrm{G} 2}=\left[-k_{2} r_{\mathrm{T}}^{2} L_{\mathrm{G} 2} / \tan \left(k_{2} L_{\mathrm{T}}\right)\right]^{1 / 2},
$$

where $k_{2}=2 \pi R 1_{\mathrm{M} 2} / c$ and $L_{\mathrm{G} 2}$ denotes the length of the glottis in M2. Table IV shows the values of $r_{\mathrm{G} 2}$ calculated from Eqs. (1) and (2) using the values of $R 1$ measured in M1 and M2 for the same pitch, vowel, and singer. Occasionally these equations yield an imaginary value for $r_{\mathrm{G} 2}$, presumably because the tract configuration was significantly different between M1 and M2; such results have been removed from the statistics. The calculated equivalent radii for most singers are in the range $2.0-2.8 \mathrm{~mm}$, the exceptions being $\mathrm{B} 1$ and T5. If these two atypical results are omitted, the average value of the effective glottal area in M2 is $19 \mathrm{~mm}^{2}$,

TABLE IV. The values of the effective glottal radius and area for falsetto register in laryngeal mechanism M2 calculated using the measured difference in $R 1$ between chest register (M1) and falsetto register (M2) when measured for the same pitch, singer, and vowel. The simple model described in the text was used. The effective glottal areas for each singer are the averages of the values calculated for each production. Calculations assumed that the radius of the tract $r_{\mathrm{T}}=15 \mathrm{~mm}$, the length of the glottis $L_{\mathrm{G} 1}=L_{\mathrm{G} 2}=10 \mathrm{~mm}$, and the effective glottal radius in $\mathrm{M} 1 r_{\mathrm{G} 1}=3.1 \mathrm{~mm}$. The glottal area in $\mathrm{M} 1$ was thus assumed to be $30.2 \mathrm{~mm}^{2}$. The average (last line) for "all" singers did not include the atypical results for singers B1 and T5.

\begin{tabular}{lcc}
\hline \hline & \multicolumn{2}{c}{ Calculated glottal parameters in falsetto register (M2) } \\
\hline Singer & Radius $(\mathrm{mm})$ & Area $\left(\mathrm{mm}^{2}\right)$ \\
\hline B1 & $1.2(1)$ & 4.5 \\
B2 & $2.0 \pm 1.3(11)$ & 18 \\
T1 & $2.4 \pm 0.8(13)$ & 20 \\
T2 & $2.8 \pm 1.1(9)$ & 28 \\
T3 & $2.3 \pm 1.3(14)$ & 21 \\
T4 & $2.0 \pm 0.4(15)$ & 14 \\
T5 & $3.6 \pm 1.0(14)$ & 44 \\
All & $2.3 \pm 1.0(62)$ & 19 \\
\hline \hline
\end{tabular}




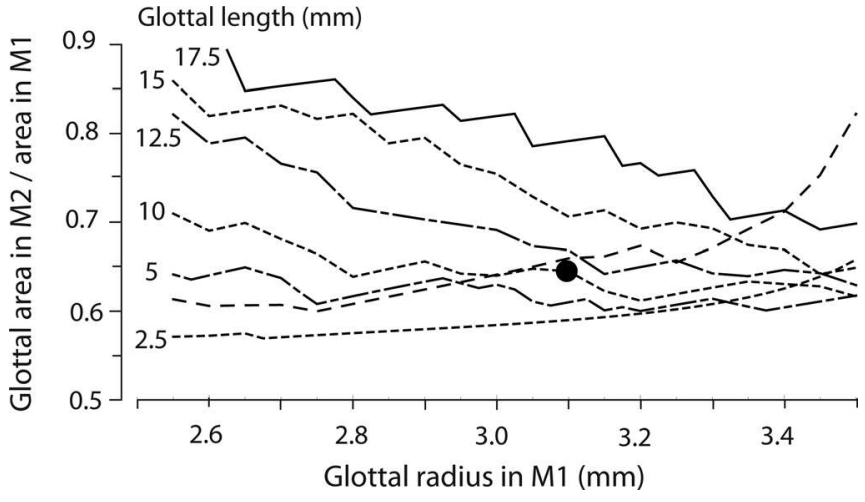

FIG. 8. The calculated ratio of the glottal area in M2 to that in M1 as a function of the glottal radius in M1. Calculations used Eqs. (1) and (2) from the simple model presented in the text and the measured differences between $R 1$ measured in chest register (M1) and falsetto register (M2) for the same singer, vowel, and pitch. Curves are presented for different values of the effective glottal length, which includes the end effects on either side, and was assumed to be equal in M1 and M2. It was assumed that the radius of the tract $r_{\mathrm{T}}=15 \mathrm{~mm}$. The black dot indicates the parameter combination used to calculate the values presented in Table IV.

compared with $30 \mathrm{~mm}^{2}$ in M1. Although the model used is a considerable simplification, this average value is in good agreement with the value of $20 \mathrm{~mm}^{2}$ reported by Hoppe et al. (2003). The model is relatively insensitive to the required assumptions-Fig. 8 demonstrates that the ratio of the effective glottal areas in M2 to M1 calculated from the experimental data is not a strong function of the assumed values of glottal radius and length in M1.

\section{CONCLUSIONS}

Comparisons made on professional male operatic singers when singing the same vowel at the same pitch, showed that the contact quotient in chest register (using laryngeal mechanism M1) exceeds that in falsetto (using M2) by typically 0.27 . The frequencies of $R 1$ and $R 2$ for $\mathrm{M} 2$ fall usually below those for M1, by typically 65 and $90 \mathrm{~Hz}$, respectively. The extent to which putative articulatory changes associated with the change in laryngeal mechanism affect resonance frequencies is unknown. However, if this is neglected, a simple model suggests that a decrease in effective glottal area by $30 \%$ to $40 \%$ is consistent with the observed decrease in resonance frequencies.

\section{ACKNOWLEDGMENTS}

We thank our volunteer subjects and the Australian Research Council for its support.

Barney, A., De Stefano, A., and Henrich, N. (2007). "The effect of glottal opening on the acoustic response of the vocal tract," Acta Acust. Acust. 93, 1046-1056.

Bunch, M., and Chapman, J. (2000). "Taxonomy of singers used as subjects in research," J. Voice 14, 363-369.

Childers, D. G., and Wong C.-F. (1994). "Measuring and modeling vocal source-tract interaction,” IEEE Trans. Biomed. Eng. 41, 663-671.

Colton, R. H. (1972). "Spectral characteristics of the modal and falsetto registers," Folia Phoniatr. 24, 337-344.

Cremer, L. (1984). The Physics of the Violin (MIT Press, Cambridge, MA), p. 253.
Delbridge, A. (1985). The Macquarie Dictionary (Macquarie Library, Dee Why, Australia), pp. 798, 809, 831.

Echternach, M., Sundberg, J., Arndt, S., Breyer, T., Mark1, M., Schumacher, M., and Richter, B. (2008). "Vocal tract and register changes analysed by real-time MRI in male professional singers-a pilot study," Logoped. Phoniatr. Vocol. 33, 67-73.

Echternach, M., Sundberg, J., Arndt, S., Mark1, M., Schumacher, M., and Richter, B. (2010). "Vocal tract in female registers-A dynamic real-time MRI study," J. Voice 24, 133-139.

Echternach, M., Sundberg, J., Markl, M., and Richter, B. (2010). "Professional opera tenors' vocal tract configurations in registers," Folia Phoniatr. Logop. 62, 278-287.

Epps, J., Smith, J. R., and Wolfe, J. (1997). “A novel instrument to measure acoustic resonances of the vocal tract during speech," Meas. Sci. Technol. 8, 1112-1121.

Fant, G., and Lin, Q. G. (1987). "Glottal source-vocal tract acoustic interaction," Speech Transmission Laboratory-Quarterly Progress and Status Report, 1/1987 (Royal Institute of Technology, Stockholm, Sweden), pp. 13-27.

Garnier, M., Henrich, N., Smith, J., and Wolfe, J. (2010). "Vocal tract adjustments in the high soprano range," J. Acoust. Soc. Am. 127, 3771-3780.

Hanna, N., Smith, J., and Wolfe, J. (2012). "Low frequency response of the vocal tract: Acoustic and mechanical resonances and their losses," Australian Acoustical Society, edited by T. McGinn, November 21-23, Fremantle, Western Australia, Australia.

Henrich, N. (2006). "Mirroring the voice from Garcia to the present day: Some insights into singing voice registers," Logoped. Phoniatr. Vocol. 31, 3-14.

Henrich, N., d'Alessandro, C., Castellengo, M., and Doval, B. (2004). "On the use of the derivative of electroglottographic signals for characterization of nonpathological phonation," J. Acoust. Soc. Am. 115, 1321-1332.

Henrich, N., d'Alessandro, C., Castellengo, M., and Doval, B. (2005). "Glottal open quotient in singing: Measurements and correlation with laryngeal mechanisms, vocal intensity, and fundamental frequency," J. Acoust. Soc. Am. 117, 1417-1430.

Henrich, N., Kiek, M., Smith, J., and Wolfe, J. (2007). "Resonance strategies used in Bulgarian women's singing style; A pilot study," Logoped. Phoniatr. Vocol. 32, 171-177.

Henrich, N., Smith, J., and Wolfe, J. (2011). "Vocal tract resonances in singing: Strategies used by sopranos, altos, tenors, and basses," J. Acoust. Soc. Am. 129, 1024-1035.

Hirano, M. (1982). "The role of the layer structure of the vocal fold in register control," Vox Humana, University of Jyvaskyla, pp. $50-62$.

Hollien, H. (1974). “On vocal registers,” J. Phonetics 2, 125-143.

Hoppe, U., Rosanowski, F., Döllinger, M., Lohscheller, J., Schuster, M., and Eysholdt, U. (2003). "Glissando: laryngeal motorics and acoustics," J. Voice 17, 370-376.

Joliveau, E., Smith, J., and Wolfe, J. (2004a). "Tuning of vocal tract resonance by sopranos," Nature 427, 116.

Joliveau, E., Smith, J., and Wolfe, J., (2004b). "Vocal tract resonances in singing: The soprano voice," J. Acoust. Soc. Am. 116, 2434-2439.

Klatt, D. H., and Klatt, L. C. (1990). "Analysis, synthesis, and perception of voice quality variations among female and male talkers," J. Acoust. Soc. Am. 87, 820-857.

Large, J., Iwata, S., and von Leden, H. (1972). "The male operatic head register versus falsetto," Folia Phoniatr. 24, 19-29.

Miller, D. G., and Schutte, H. K. (2005). "Mixing the registers: Glottal source or vocal tract?," Folia Phoniatr. Logop. 57, 278-291.

Miller, D. G., Sulter, A. M., Schutte, H. K., and Wolf, R. F. (1997). "Comparison of vocal tract formants in singing and non-periodic phonation," J. Voice 11, 1-11.

Neumann, K., Schunda, P., Hoth, S., and Euler, H. A. (2005). "The interplay between glottis and vocal tract during the male passaggio," Folia Phoniatr. Logop. 57, 308-327.

Nord, L., Ananthapadmanabha, T. V., and Fant, G. (1986). "Signal analysis and perceptual tests of vowel responses with an interactive source-filter model," J. Phonetics 14, 401-404.

Rothenberg, M. (1980). Acoustic Interaction between the Glottal Source and the Vocal Tract, edited by K. N. Stevens and M. Hirano (University of Tokyo Press, Tokyo), pp. 305-328. 
Roubeau, B. (1993). "Mécanismes vibratoires laryngés et contrôle neuromusculaire de la fréquence fondamentale" ("Laryngeal vibratory mechanisms and neuro-muscular control of fundamental frequency"), Ph.D. thesis, Université Paris-Orsay, Orsay.

Roubeau, B., Castellengo, M., Bodin, P., and Ragot, M. (2004). "Phonétogramme par mécanisme laryngé" ("Laryngeal registers as shown in the voice range profile") Folia Phoniatr. Logop. 56, 321-333.

Roubeau, B., Henrich, N., and Castellengo, M. (2009). "Laryngeal vibratory mechanisms: The notion of vocal register revisited," J. Voice 23, 425-438.

Salomão, G. L., and Sundberg, J. (2009). "What do male singers mean by modal and falsetto register? An investigation of the glottal voice source," Logoped. Phoniatr. Vocol. 34, 73-83.

Smith, J. R. (1995). "Phasing of harmonic components to optimize measure signal-to-noise ratios of transfer functions," Meas. Sci. Technol. 6, 1343-1348.

Sundberg, J. (1975). "Formant technique in a professional female singer," Acustica 32, 89-96.
Sundberg, J., and Högset, C. (2001). "Voice source differences between falsetto and modal registers in counter tenors, tenors and baritones," Logoped. Phoniatr. Vocol. 26, 26-36.

Sundberg, J., La, F. M. B., and Gill, B. P. (2011). "Professional male singers' formant tuning strategies for the vowel /a/," Logoped. Phoniatr. Vocol. 36, 156-167.

Sundberg, J., and Nordström, P.-E. (1983). "Raised and lowered larynx: The effect on vowel formant frequencies," J. Res. Singing 6, 7-15.

Swerdlin, Y., Smith, J., and Wolfe, J. (2010). "The effect of whisper and creak vocal mechanisms on vocal tract resonances," J. Acoust. Soc. Am. 127, 2590-2598.

Titze, I. R. (2004). "Theory of glottal airflow and source-filter interaction in speaking and singing," Acta Acust. Acust. 90, 641-648.

Titze, I. R. (2008). "Nonlinear source-filter coupling in phonation: Theory," J. Acoust. Soc. Am. 123, 2733-2749.

Wolfe, J., Garnier, M., and Smith, J. (2009). "Vocal tract resonances in speech, singing and playing musical instruments," Human Frontier Sci. Progr. J. 3, 6-23. 